\title{
Placing Dental Implants through Impacted Teeth to Support a Fixed Partial Denture in a Geriatric Patient as an Alternative to Invasive Extraction Surgeries
}

\author{
Jorge Cortés-Bretón Brinkmann¹ Marina Lobato-Peña² Fabián Pérez-González¹ \\ Pedro Molinero-Mourelle ${ }^{1}$ Luis Sánchez-Labrador ${ }^{1} \quad$ Juan Santos-Marino $^{3}$ Juan López-Quiles ${ }^{1}$ \\ Jose María Martínez-González ${ }^{1}$
}

1Department of Dental Clinical Specialties, Faculty of Dentistry,
Complutense University of Madrid, Madrid, Spain
2Department of Surgery and Medical-Surgical Specialities,
Area of Orthodontics, University Medical and Dental School,
University of Oviedo, Oviedo, Spain
${ }^{3}$ Department of Surgery, University of Salamanca, Salamanca, Spain

Address for correspondence Jorge Cortés-Bretón Brinkmann, DDS, PhD, Department of Dental Clinical Specialties, School of Dentistry, Complutense University of Madrid, Pza Ramon y Cajal s/n, Madrid 28040, Spain (e-mail: brinkmann55@hotmail.com).

Eur J Dent:2020;14:697-701

\begin{abstract}
Keywords

- dental implants

- impacted teeth

- invasive extraction surgery

- alternative

- therapeutic option

- elderly patients

Multiple dental impactions not associated with craniofacial syndromes are a rare condition and present the dentist with a therapeutic challenge when it comes to performing surgical/restorative treatments in adult patients. This case report describes a geriatric patient with multiple impacted teeth restored by means of two different protocols. In the second quadrant, an impacted tooth was extracted followed by regeneration and placement of an implant. In the third quadrant, implants were placed though impacted teeth for restoration with a fixed partial prosthesis. Placing dental implants through impacted teeth may offer a possible therapeutic option for implant-supported restorations in middle-aged or elderly patients, for whom surgery and orthodontic traction are not possible, and/or patients who refuse to undergo more invasive extraction surgery.
\end{abstract}

\section{Introduction}

When planning treatments involving dental implants, dentists occasionally come across patients presenting root remains and/or impacted teeth in the area where an implant is to be placed. This clinical situation can be solved in various ways. First, impacted teeth may be moved to a more favorable position by orthodontic traction. This option has been reported in teenage patients and those aged under 30 years. ${ }^{1}$ Second, a surgical approach can be planned that avoids infringing the impacted tooth or root fragment, by inclining the implant or placing it on the vestibular or palatine/lingual side of the impacted tooth. ${ }^{2}$ Another option described in the literature is to extract the impacted tooth or root fragment followed by guided tissue regeneration. This is considered the gold standard approach to the problem when treating adult patients. ${ }^{3}$ But this option is more invasive and treatment takes longer. Finally, the use of short or extra-short implants offers another means of avoiding interference with an impacted tooth. ${ }^{4}$

Ever since Branemark discovered the phenomenon of osteointegration in the 1950s, direct bone-to-implant surface contact has been considered the principal means of evaluating healing responses around endosseous implants. Osteointegrated implants are rigidly connected through intimate contact of bone tissue to the implant suface. ${ }^{5}$ The entire process involves a complex array of events including the formation of a provisional fibrin matrix, which is organized by blood vessels, collagen fibers, and trabecular bone, subsequently maturating into lamellar bone and bone marrow. ${ }^{6,7}$

In 2009, Davarpanah and Szmukler-Moncler ${ }^{8}$ published the first of a series of articles ${ }^{9-12}$ about a paradigmatic change when it comes to drilling and placing implants intentionally in contact with dental tissues, remaining root fragments or impacted teeth. The protocol achieved success rates similar to conventional implant placement in the medium term. 
Based on this principle, inserting implants through impacted teeth would appear to offer a potential treatment alternative. ${ }^{13}$

This clinical case report describes a geriatric patient with multiple impacted teeth without any associated syndromes, who was rehabilitated by means of two different protocols. In the second quadrant, the impacted tooth was dealt with by the classic approach for middle-aged or elderly patients, which consists of extraction of the tooth with simultaneous regeneration and implant placement. In the third quadrant, implants were placed through the impacted teeth for rehabilitation with a fixed denture. As far as the authors are aware, this is the first case report that describes the use of this alternative approach in a patient with multiple vertically positioned impacted teeth.

\section{Case Report}

A female patient, aged 78 years came to our clinic in September 2017 for the extraction of deciduous teeth in the third quadrant (lower deciduous canine and two molars). The patient classified as ASA I (according to the American Society of Anesthesiologists Physical Status Classification System) was not taking any medication and was not allergic to any drug. She did not smoke or have any other addictive habits. Clinical examination found deciduous teeth remaining in adulthood in the third quadrant with pronounced mobility (-Fig. 1A), which were causing pain. Radiographs showed the presence of multiple impacted teeth in both jaws with the presence of deciduous teeth in the lower left quadrant (canine and molars). Impactions were observed of the upper right canine, lower left canine, as well as the two lower left premolars (-Fig. 1B).

This patient had attended the clinic 8 years earlier (in April 2009) for the extraction of a deciduous upper left canine, together with the simultaneous extraction of the palatally retained canine and corresponding regeneration by means of a fine particulate xenograft (Apatos, Osteógenos, Madrid, Spain). An implant (external hexagon, 3.8×10 mm Defcon $\mathrm{TSH}{ }^{\circledR}$, Phibo, Barcelona, Spain) was placed in the same surgical session. Although the clinical outcome of the implantsupported crown after 8 years was acceptable and had never caused any problems (-Fig. 2A, 2B), the patient had been traumatized by the surgery and postoperative period and refused to undergo similar surgery in the mandible.

For this reason, it was proposed to place implants anchored in the impacted teeth. The treatment protocol planned fulfilled Declaration of Helsinki guidelines for experimentation involving human subjects. The procedure was explained to the patient, as well as the risks and possible complications involved, and she gave her informed consent to proceed. It should be noted that no change in the positions of the impacted teeth was observed since the first time the patient had attended the clinic 8 years earlier ( - Fig. 3 ).

Following extraction of the deciduous teeth, two external hex implants (S.I.N. Implant system, Sao Paulo, Brazil) of $10 \mathrm{~mm}$ length were placed in position of the canine $(3.75 \mathrm{~mm}$ diameter) and second premolar (4.1 mm diameter). The procedure was performed under local anesthetic; the implants were inserted through the impacted teeth at the canine and
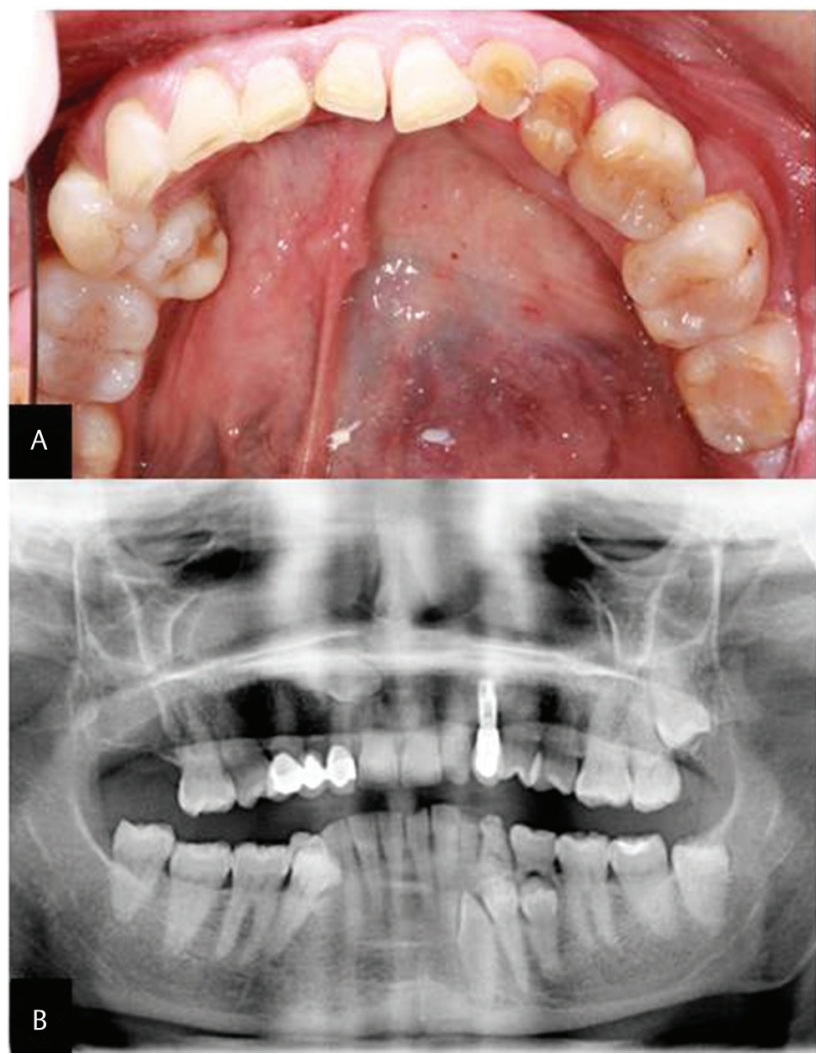

Fig. 1 (A) Clinical image of third quadrant, showing deciduous teeth: canine and first and second molars. (B) Panoramic radiograph of patient showing multiple impacted teeth, one canine in the first quadrant, and one canine and two premolars in the third quadrant. It is also noted an implant placed in second quadrant.

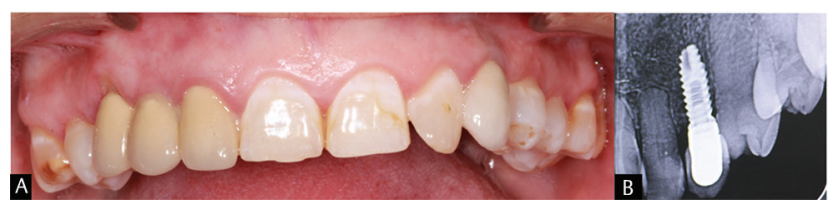

Fig. 2 (A) Clinical image shows acceptable clinical outcome of the implant-supported crown in the upper left quadrant in position of the canine 8 years after implant placement. (B) Periapical radiograph of the implant-crown complex in canine position in second quadrant, exhibiting no bone loss.

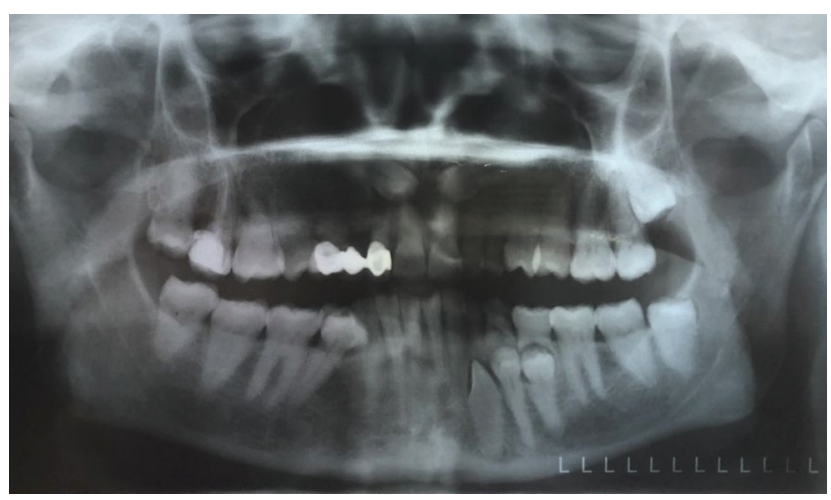

Fig. 3 Panoramic radiograph of the patient 8 years before. No significant changes in the positions of impacted teeth have taken place up to the present. 


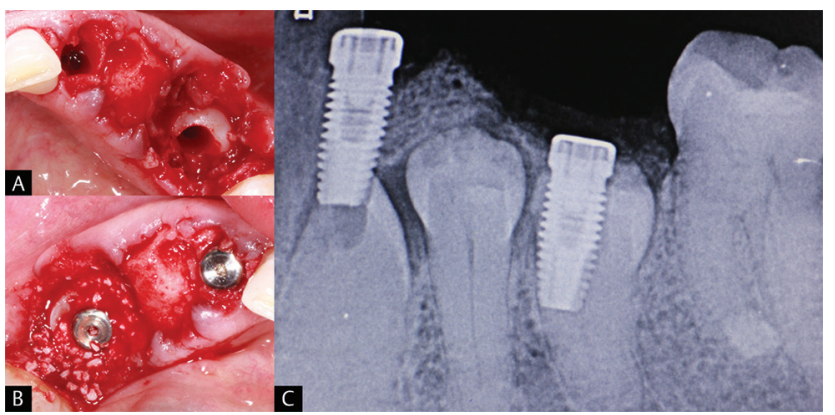

Fig. 4 (A) Clinical image after extracting deciduous teeth, finishing drilling sequence through impacted teeth. (B) Clinical image of implants anchored through impacted teeth after regenerating the gap with particulate xenograft. (C) Postsurgical periapical radiograph showing implants placed through impacted teeth.

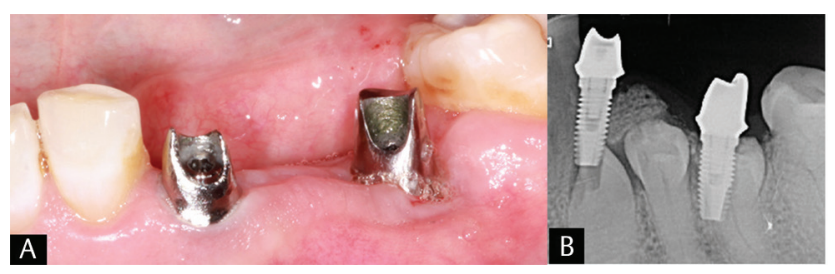

Fig. 5 (A) Clinical image of individualized abutments screwed to implants; image shows good gingival health. (B) Periapical radiograph verifying correct fit of individualized abutments.

second premolar positions. It was necessary to follow the complete drilling sequence with abundant irrigation because of the hardness of the dental tissues ( - Fig. 4A). A single dehiscence defect and the exposed threads of the implant were grafted with fine grain xenograft (Apatos, Osteógenos) ( - Fig. 4B) without any membrane; the surgical sites were sutured with $5 / 0$ polyamide multifilament suture (Supramid, Aragó, Barcelona, Spain).

The postsurgical periapical radiograph showed implant placement in the second premolar position completely passing through the tooth, while the implant in canine position penetrated the coronal part of the impacted tooth ( $\sim$ Fig. 4C). No postoperative pain was recorded and both implants placed through the impacted teeth healed uneventfully. Sutures were removed 10 days after surgery and rigorous follow-up of the patient continued throughout the healing period, with follow-up visits scheduled after 1 month and 2 months.

Four months after surgery the second phase commenced, connecting healing abutments. Fifteen days later, the restoration phase began, consisting of an implant-supported metal ceramic denture. To improve the prosthetic crown emergence, individualized screw-retained abutments were used to support the prosthesis ( $\boldsymbol{- F i g}$. 5A, B). The restoration was cemented onto the abutments ( $\mathbf{- F i g .} \mathbf{6 A}, \mathbf{6 B}$ ) using zinc oxide-based provisional cement with eugenol (Temp-Bond Original; Kerr Dental, Detroit, United States).

At follow-up visits, the case evolved satisfactorily, fulfilling the success criteria established by Buser et $\mathrm{al}^{14}$ and Cochran et $a l .{ }^{15}$ No radiolucent images around the implants were observed in radiographs taken during the follow-up, nor were any abnormal reactions at the bone-to-implant

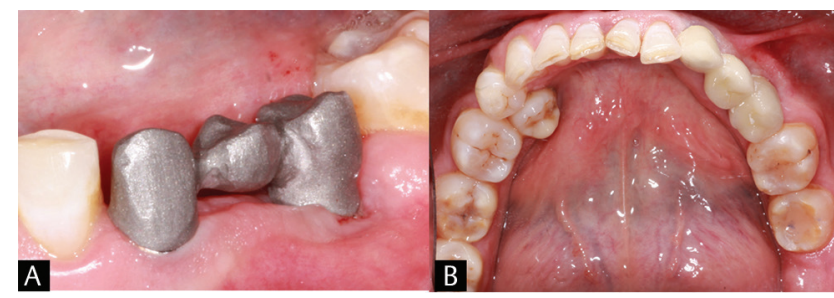

Fig. 6 (A) Clinical image: checking metal prosthesis on individualized abutments. (B) Occlusal view of fixed partial denture.

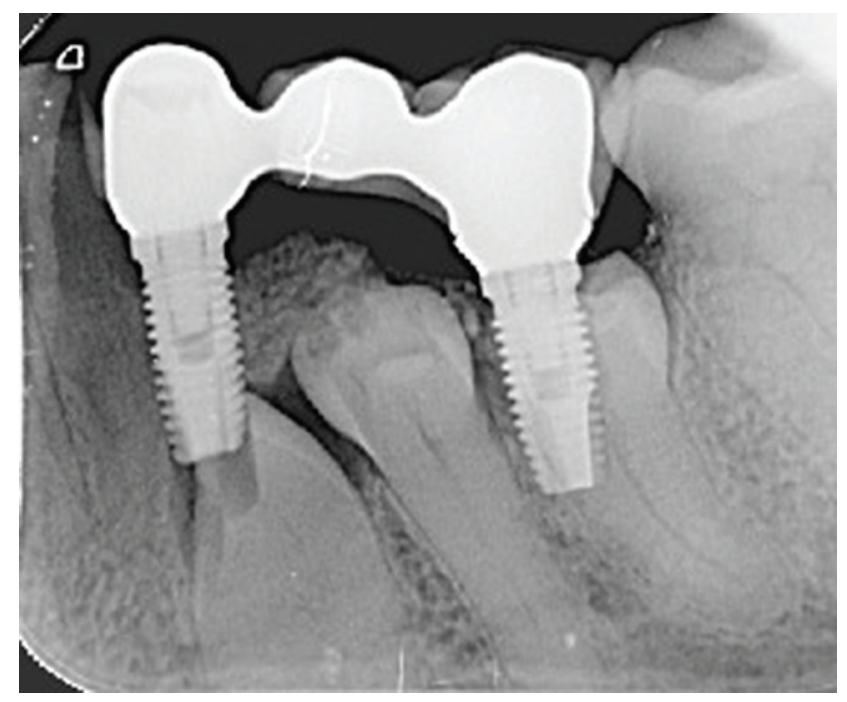

Fig. 7 Periapical radiograph after 24 months follow-up. Neither impacted teeth nor implants have undergone any changes.

or impacted tooth-to-implant interfaces. The patient was recalled every 6 months after delivery of the definitive restoration, undergoing clinical and radiographic examination ( - Fig. 7), and to date (a follow-up period of 24 months), no signs or symptoms of failure have occurred, or any complication at any stage.

The patient found treatment acceptable and was pleased to have avoided the trauma of extraction.

\section{Discussion}

Dental impaction has been reported to affect as much as 25 to $50 \%$ of the population..$^{16}$ Multiple impacted teeth not associated with craniofacial syndromes are a rare condition and present a therapeutic challenge to the dentist. ${ }^{17}$ The first option for teenagers and young adults is a combination of surgery and orthodontic traction to move the impacted teeth into position. In this context, Becker and Chaushu ${ }^{1}$ made a study of palatally impacted canines, concluding that the prognosis for successful orthodontic resolution of an impacted canine in an adult is poorer than in a younger patient and that prognosis worsens with age. Furthermore, when this treatment is performed in older patients, a successful outcome may be expected to take considerably longer.

For this reason, cases of impacted teeth are often approached through surgery and subsequent restoration. In 
this sense, extraction of an impacted tooth with simultaneous implant placement and regeneration are considered the "gold standard" treatment. ${ }^{3}$ But this approach is more invasive and prolongs treatment, particularly in cases where cortical bone is not adequately preserved, and so it may not be possible to place implants during the same surgical session. Consequently, this option is often refused by patients.

Notwithstanding as a rule, clinicians tend to prefer to place implants in pristine and/or regenerated bone. As affirmed by Melcher, ${ }^{18}$ the characteristics of wound healing are determined by the type of cells that repopulate the lesioned area. When drilling bone, the cells that occupy the lesion comprise bone cells, bone marrow, and blood cells, which will determine the healing pattern and the formation of direct bone apposition over the titanium implant surface. However, diverse experimental histological animal studies have suggested the possibility of achieving implant anchorage within or encroaching on ankylosed root remains or impacted teeth with periodontium simulating that found around natural teeth. ${ }^{19-21}$ Microscope studies have affirmed the appearance of periodontal ligament with a layer of cementum over the implant surface occupying a space containing the collagen fibers and blood vessels characteristic of periodontal ligament. ${ }^{20,21}$

On the basis of these findings, Davarpanah and SzmuklerMoncler $^{8-12}$ published a series of articles (the first in 2009) describing a case series in which implants were placed through ankylosed impacted root remains or impacted teeth. The authors suggested that this alternative to the conventional bone-to-implant interface would not interfere with the implants or their mid- to long-term survival.

Nevertheless, recent articles by Nevins et $\mathrm{al}^{22}$ and Langer et $\mathrm{a}^{23}$ reported belated implant failures when placed through impacted root remains. Scanning electron microscopic evaluation revealed that the failed implant surfaces were infiltrated by bacterial deposits and calculus. The authors recommend caution when placing implants through retained root fragments, as this involves long-term risk. But the case series published by Amato et $\mathrm{al}^{24}$ and Davarpanah et $\mathrm{al}^{9,12}$ who placed implants through impacted teeth, obtained satisfactory results in the medium term. This difference in prognosis could be due to the fact that root remains were in contact with the buccal medium for a long time, involving foreseeable bacterial penetration, which could lead to failed implant osteointegration. Besides the impacted teeth are not related with infection issue, whereas the retained roots might be.

These procedures aim to shorten restorative treatment time and are more acceptable to the patient. As they maintain the integrity of the vestibular table, they also optimize esthetic outcomes as remaining crown fragments provide greater support for soft tissues. ${ }^{25}$

The protocol described in this case report is unconventional and not extensively documented. Nevertheless, it offers a useful treatment option that avoids the trauma of extraction and reduced treatment time in cases in which the outcomes may in any case be unpredictable.

\section{Conclusion}

Within the limitations of this case report, placing dental implants through impacted teeth would appear to offer a possible therapeutic option for implant-supported restorations in middle-aged or elderly patients, for whom surgery and orthodontic traction are not possible, and/or patients who refuse to undergo more invasive extraction surgery. Further studies are needed with longer follow-ups, larger sample sizes, and standardized clinical protocols to confirm the outcomes of the present case report, before this protocol can be introduced into routine clinical therapeutic practice.

\section{Conflict of Interest}

None declared.

\section{References}

1 Becker A, Chaushu S. Success rate and duration of orthodontic treatment for adult patients with palatally impacted maxillary canines. Am J Orthod Dentofacial Orthop 2003;124(5):509-514

2 Mazor Z, Segal P, Levin L. Computer-guided implant placement for rehabilitation of the edentulous maxilla with two impacted canines: an approach without extraction of the impacted teeth. Int J Periodontics Restorative Dent 2015;35(1):93-97

3 García B, Boronat A, Larrazabal C, Peñarrocha M, Peñarrocha M. Immediate implants after the removal of maxillary impacted canines: a clinical series of nine patients. Int J Oral Maxillofac Implants 2009;24(2):348-352

4 Felice P, Barausse C, Stefanini M, Pistilli R, Zucchelli G. A minimally invasive approach using a 4 -mm implant without extraction of impacted maxillary canine: four-year postloading results. Int $\mathrm{J}$ Periodontics Restorative Dent 2017;37(6):819-824

5 Schroeder A, van der Zypen E, Stich H, Sutter F. The reactions of bone, connective tissue, and epithelium to endosteal implants with titanium-sprayed surfaces. J Maxillofac Surg $1981 ; 9(1): 15-25$

6 Berglundh T, Abrahamsson I, Lang NP, Lindhe J. De novo alveolar bone formation adjacent to endosseous implants. Clin Oral Implants Res 2003;14(3):251-262

7 Schwarz F, Herten M, Sager M, Wieland M, Dard M, Becker J. Histological and immunohistochemical analysis of initial and early osseous integration at chemically modified and conventional SLA titanium implants: preliminary results of a pilot study in dogs. Clin Oral Implants Res 2007;18(4):481-488

8 Davarpanah M, Szmukler-Moncler S. Unconventional implant treatment: I. Implant placement in contact with ankylosed root fragments. A series of five case reports. Clin Oral Implants Res 2009;20(8):851-856

9 Davarpanah M, Szmukler-Moncler S. Unconventional implant placement. 2: placement of implants through impacted teeth. Three case reports. Int J Periodontics Restorative Dent 2009; 29(4):405-413

10 Szmukler-Moncler S, Davarpanah M, Davarpanah K, Capelle-Ouadah N, Demurashvili G, Rajzbaum P. Unconventional implant placement part III: implant placement encroaching upon residual roots - a report of six cases. Clin Implant Dent Relat Res 2015;17(Suppl 2) :e396-e405

11 Szmukler-Moncler S, Davarpanah M, Davarpanah K, Rajzbaum P, Capelle-Ouadah N, Demurashvili G. [Implants in contact with tissues other than bone. Is there room for a potential paradigm shift?]. Swiss Dent J 2014;124(2):149-164

12 Davarpanah M, Szmukler-Moncler S, Rajzbaum P, Davarpanah K, Capelle-Ouadah N, Demurashvili G. 
Unconventional implant placement. V: implant placement through impacted teeth; results from 10 cases with an 8- to 1-year follow-up. Int Orthod 2015;13(2):164-180

13 Labidi A, Bekri S, Mansour L, Ghoul-Mazgar S. Implants placement in contact with dental tissue: a potential paradigm shift? Systematic literature review. Eur J Dent 2019;13(4):642-648

14 Buser D, Mericske-Stern R, Bernard JP, et al. Long-term evaluation of non-submerged ITI implants. Part 1: 8-year life table analysis of a prospective multi-center study with 2359 implants. Clin Oral Implants Res 1997;8(3):161-172

15 Cochran DL, Buser D, ten Bruggenkate CM, et al. The use of reduced healing times on ITI implants with a sandblasted and acid-etched (SLA) surface: early results from clinical trials on ITI SLA implants. Clin Oral Implants Res 2002;13(2):144-153

16 Andreasen JO, Pindborg JJ, Hjörting-Hansen E, Axéll T. Oral health care: more than caries and periodontal disease. A survey of epidemiological studies on oral disease. Int Dent J 1986;36(4):207-214

17 Bayar GR, Ortakoglu K, Sencimen M. Multiple impacted teeth: report of 3 cases. Eur J Dent 2008;2(1):73-78

18 Melcher AH. On the repair potential of periodontal tissues. J Periodontol 1976;47(5):256-260

19 Gray JL, Vernino AR. The interface between retained roots and dental implants: a histologic study in baboons. J Periodontol 2004;75(8):1102-1106
20 Parlar A, Bosshardt DD, Unsal B, Cetiner D, Haytaç C, Lang NP. New formation of periodontal tissues around titanium implants in a novel dentin chamber model. Clin Oral Implants Res 2005;16(3):259-267

21 Ruggeri A, Franchi M, Marini N, Trisi P, Piatelli A. Supracrestal circular collagen fiber network around osseointegrated nonsubmerged titanium implants. Clin Oral Implants Res 1992;3(4):169-175

22 Nevins ML, Langer L, Schupbach P. Late dental implant failures associated with retained root fragments: case reports with histologic and SEM analysis. Int J Periodontics Restorative Dent 2018;38(1):9-15

23 Langer L, Langer B, Salem D. Unintentional root fragment retention in proximity to dental implants: a series of six human case reports. Int J Periodontics Restorative Dent 2015;35(3):305-313

24 Amato F, Macca U, Amato G, Mirabella D. Immediate loading of implants inserted through impacted teeth in the esthetic area: a series of 10 cases with up to 7 years of follow-up. Int J Periodontics Restorative Dent 2019;39(3):325-332

25 Hürzeler MB, Zuhr O, Schupbach $P$, Rebele SF, Emmanouilidis N, Fickl S. The socket-shield technique: a proof-of-principle report. J Clin Periodontol 2010;37(9): 855-862 